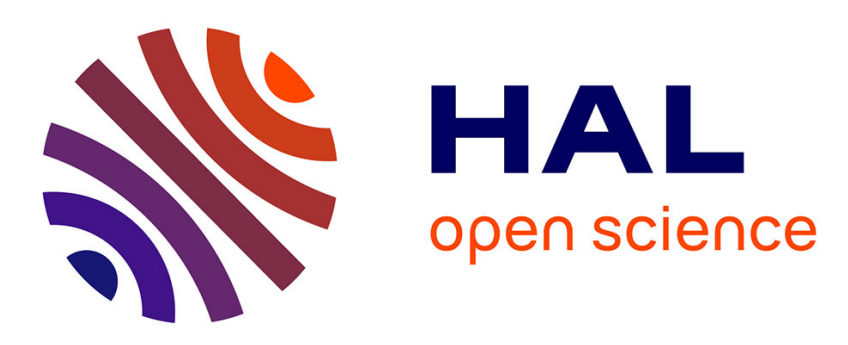

\title{
Lait, sang, larmes en offrande : la manipulation des fluides corporels féminins comme support d'une élaboration éthique pour la biosphère
}

\author{
Marine Legrand, Anaïs Tondeur
}

\section{- To cite this version:}

Marine Legrand, Anaïs Tondeur. Lait, sang, larmes en offrande : la manipulation des fluides corporels féminins comme support d'une élaboration éthique pour la biosphère. Journal international de bioéthique et d'éthique des sciences , 2019, 30 (4), pp.19-49. 10.3917/jibes.304.0019 . hal-02504559

\author{
HAL Id: hal-02504559 \\ https://hal.science/hal-02504559
}

Submitted on 26 Jun 2020

HAL is a multi-disciplinary open access archive for the deposit and dissemination of scientific research documents, whether they are published or not. The documents may come from teaching and research institutions in France or abroad, or from public or private research centers.
L'archive ouverte pluridisciplinaire HAL, est destinée au dépôt et à la diffusion de documents scientifiques de niveau recherche, publiés ou non, émanant des établissements d'enseignement et de recherche français ou étrangers, des laboratoires publics ou privés. 


\begin{tabular}{|c|c|}
\hline $\begin{array}{l}\text { Titre } \\
\text { Title }\end{array}$ & $\begin{array}{l}\text { Lait, sang, larmes en offrande : la manipulation des fluides } \\
\text { corporels féminins comme support d'une élaboration éthique pour la } \\
\text { biosphère }\end{array}$ \\
\hline $\begin{array}{l}\text { Autrices } \\
\text { Authors }\end{array}$ & $\begin{array}{l}\text { Marine Legrand } \\
\text { Anaïs Tondeur }\end{array}$ \\
\hline $\begin{array}{l}\text { Affiliations } \\
\text { Affiliations }\end{array}$ & $\begin{array}{l}{ }^{1} \text { Laboratoire Eau, Environnement et Systèmes urbains - LEESU, } \\
\text { Ecole des Ponts Paris Tech, Marne-la-vallée (France) } \\
{ }^{2} \text { Artiste (France) }\end{array}$ \\
\hline $\begin{array}{l}\text { Autrice de } \\
\text { correspondance } \\
\text { Corresponding author }\end{array}$ & $\begin{array}{l}\text { Marine Legrand : marine.m.legrand@gmail.com ; } 66 \text { rue des } \\
\text { Rondeaux } 75020 \text { Paris, France }\end{array}$ \\
\hline $\begin{array}{l}\text { Résumé FR ( } 250 \text { mots }) \\
\text { Abstract FR (250 words) }\end{array}$ & $\begin{array}{l}\text { L'empreinte toujours croissante des activités humaines sur les } \\
\text { milieux de vie conduit des acteurs de tout ordre à chercher à } \\
\text { développer de nouvelles éthiques environnementales, qui passent } \\
\text { tant par l'élaboration théorique que par l'expérimentation. Celles-ci } \\
\text { diffèrent dans leurs fondements comme dans leurs conséquences } \\
\text { pratiques, en ce qu'elles mettent l'accent sur la vie de chaque être, } \\
\text { les écosystèmes, les processus évolutifs, ou bien encore l'intérêt } \\
\text { bien compris de notre propre espèce. Choisissant d'entremêler } \\
\text { expérimentation et théorisation au travers d'une exploration hybride } \\
\text { entre art contemporain et anthropologie, nous avons mené une série } \\
\text { de performances impliquant nos propres fluides corporels féminins } \\
\text { ou considérés comme tels - lait maternel, sang menstruel, larmes du } \\
\text { deuil - dont nous avons fait des offrandes à différents milieux. Ces } \\
\text { gestes, conçus comme des rituels, participent d'une mise en } \\
\text { discussion, avec différents publics, du dialogue entre existences } \\
\text { humaines et cycles biogéochimiques. Celle-ci poursuit, tout en les } \\
\text { dépassant, les analogies anciennes entre fertilité féminine et } \\
\text { terrestre. Nous constatons qu'une piste s'ouvre ainsi, s'appuyant sur } \\
\text { une pensée renouvelée de la physiologie, vers une approche éthique } \\
\text { particulière tournée vers la biosphère : elle puise dans les } \\
\text { continuités matérielles et symboliques entre les existences humaines } \\
\text { et le reste du monde vivant, partant de ce qu'il y a de plus intime en } \\
\text { chacune et chacun de nous. }\end{array}$ \\
\hline $\begin{array}{l}\text { Résumé ENG (250 } \\
\text { mots) } \\
\text { Abstract ENG }(250 \\
\text { words })\end{array}$ & $\begin{array}{l}\text { The ever-growing ecological imprint of human activities has lead } \\
\text { numerous and diverse actors to develop new environmental ethical } \\
\text { approaches, both based on experimentation and theoretical } \\
\text { elaboration. These approaches hold multiple philosophical roots and } \\
\text { practical consequences: some insist on the life and value of each } \\
\text { being, ecosystems or evolutional processes, other insist on the } \\
\text { instrumental value of nature. As for us, we have chosen to bring } \\
\text { together experimentation and theorization, in order to lead an hybrid } \\
\text { exploration between art and anthropology. Since } 2015 \text {, we have }\end{array}$ \\
\hline
\end{tabular}




\begin{tabular}{|c|c|}
\hline & $\begin{array}{l}\text { conducted a series of performances, stemming from our own body } \\
\text { fluids, considered as feminine (breastmilk, menstrual blood, tears of } \\
\text { grief), and used as offerings for different living environments (« } \\
\text { milieux de vie »). These gestures, conceived as rituals, led to debate } \\
\text { with different publics about the relation between human existence } \\
\text { and biogeochimical cycles. This reflection is grounded in the } \\
\text { analogies between terrestrial and female fertility, but it goes one } \\
\text { step further. We propose that a new pathway could be opened, } \\
\text { based on a renewal of physiological concepts in relation with the } \\
\text { development of an ethical position for the protection of the } \\
\text { biosphere. It is rooted in the intimacy of each of us, and the material } \\
\text { and symbolic continuities between human existence and the rest of } \\
\text { the living word. }\end{array}$ \\
\hline $\begin{array}{l}\text { Nombre de mots de } \\
\text { l'article (5000-10 000) } \\
\text { Article words count } \\
(5000-10000)\end{array}$ & 9849 mots \\
\hline $\begin{array}{l}\text { Mots-clés FR } \\
\text { Keywords FR }\end{array}$ & $\begin{array}{l}\text { Ethique environnementale, écoféminisme, rituel, physiologie, } \\
\text { fluides corporels, biosphère }\end{array}$ \\
\hline $\begin{array}{l}\text { Mots-clés ENG } \\
\text { Keywords ENG }\end{array}$ & $\begin{array}{l}\text { Environmental ethics, eco-feminism, ritual, physiology, body fluids, } \\
\text { biosphere }\end{array}$ \\
\hline $\begin{array}{l}\text { Financement du projet } \\
\text { Funding of the project }\end{array}$ & $\begin{array}{l}\text { Ce projet a été en partie financé dans le cadre de la résidence Sols } \\
\text { Fictions (COAL/Domaine de Chamarande) }\end{array}$ \\
\hline $\begin{array}{l}\text { Conflits d'intérêts } \\
\text { Conflicts of interests }\end{array}$ & RAS \\
\hline $\begin{array}{l}\text { Remerciements } \\
\text { Acknowledgements }\end{array}$ & $\begin{array}{l}\text { Les membres du collectif Chaoïde - Alan Vergnes, Germain } \\
\text { Meulemans, Yesenia Thibault Picazo - ainsi que Nathalie Blanc \& } \\
\text { Maëva Blandin, Lauranne Germond et Loïc Fel pour le Laboratoire } \\
\text { de la culture durable ; Joanne Clavel pour notre travail commun } \\
\text { autour de l'Appel aux larmes ; les visiteurs de l'exposition SOLS } \\
\text { FICTIONS, les organisateurs des différents évènements qui ont } \\
\text { accompagné la création du triptyque et participants à ces } \\
\text { expériences : présentation LASER, Annick Bureaud ; séminaire « } \\
\text { méthodes sensorielles et visuelles en anthropologie » EHESS (en } \\
\text { particulier Florencia Muñoz Ebensperger et Léo Nguyen Van Thé) } \\
\text {; Perig Pitou et les membres du colloque «La vie à l'œuvre » au } \\
\text { Musée de la Chasse et de la Nature ; les participants à la } \\
\text { performance L'APPEL AUX LARMES au centre Tignous pour } \\
\text { l'art contemporain (en particulier Hélène, Laure, Marianne, Sidonie } \\
\text { \& Cécile) }\end{array}$ \\
\hline $\begin{array}{l}\text { Références au style } \\
\text { Vancouver } \\
\text { Vancouver style } \\
\text { references }\end{array}$ & $\begin{array}{l}\text { [1] d'Eaubonne, F. (1974) Le féminisme ou la mort, Paris : Éd. A. } \\
\text { Moreau, } 228 \text { p. } \\
\text { [2] d'Eaubonne F. (1978), Écologie, féminisme : révolution ou } \\
\text { mutation? Paris : Ed. ATP, 223p. }\end{array}$ \\
\hline
\end{tabular}


[3] Gandon, A. (2009), L'écoféminisme : une pensée féministe de la nature et de la société, Recherches féministes, 22(1), 5-25.

[4] Hache, E. (2016), Reclaim, recueil de textes écoféministes. Ed. Cambourakis, $416 \mathrm{p}$.

[5] Meulemans G., Legrand M., Tondeur A. Thibault-Picazo Y., Vergnes A. (2017), Soil fictions: addressing urban soils between art, soil ecology and anthropology, Collaborative Anthropologies, 10(1), 20-44.

[6] Merchant, C. (1980), The Death of Nature : Women, Ecology, and Scientific Revolution. New-York : HarperOne, 384 p.

[7] Callicott, J. B. (1985), Intrinsic value, quantum theory, and environmental ethics, Environmental ethics, 7(3), 257-275.

[7] Hache, E. (2011), Ce à quoi nous tenons. Propositions pour une écologie pragmatique, Paris : La découverte, 216.

[8] Viveiros de Castro, E. (2009), Métaphysiques cannibales : lignes d'anthropologie post-structurale, Paris : PUF, $216 \mathrm{p}$. [9] Holgate, M. (1999), The Green Web, a Union for World Conservation, Londres : Earthscan Publications Ltd, 308 p. [10] Blandin, P. (2009), De la protection de la nature au pilotage de la biodiversité, Versailles : Quae, 13 et suiv.

[11] Afeissa, S. (2009), Ethique de l'environnement, Paris : VRIN ; 53-58.

[12] Leopold A. A Sand County Almanac, New York : Oxford University Press : 1949.

[13] Prigogine I. \& Stengers I. (1979), La nouvelle alliance : métamorphose de la science, Paris : Gallimard, $312 \mathrm{p}$.

[14] Callicott, J. B.(1985), op.cit.

[15] Larrère C \& Larrère R. (2009), Du bon usage de la nature : pour une philosophie de l'environnement, Paris : Flammarion, 105-164.

[16] Frankel O., Soulé M. E. (1981) Conservation and evolution, Cambridge : CUP Archive, 327 p.

[18] Gorz, A. \& Bosquet, M. (1978), Ecologie et politique, Paris : Seuil, 256 p.

[19] Ellul, J. (1977), Le système technicien, Paris : Calmann-Lévy, $344 \mathrm{p}$.

[20] Castoriadis, C. (1999 (1961)), L'institution imaginaire de la société, Paris : Le Seuil, 544 p.

[21] d'Eaubonne, F. (1976), Les femmes avant le patriarcat, Paris : Payot, $239 \mathrm{p}$.

[22] Foster, J.B. (2000), Marx's ecology. Materialism and Nature, New York: Monthly Review Press, 200 p.

[23] Lowy, M. (2011), Écosocialisme : l'alternative radicale à la catastrophe écologique capitaliste, Paris : Fayard/Mille et une nuits, $240 \mathrm{p}$.

[24] Fressoz, J.B. (2011), Ecologies marxistes et écologies de la modernité. À propos de John Bellamy Foster, Marx's ecology.

Materialism and Nature, Monthly. Review Press, New York, 2000, Mouvements, 66, 155 - 159. 
[25] Cauvin, J. (1994), Naissance des divinités, naissance de l'agriculture : la révolution des symboles au néolithique, Paris :

CNRS Editions, 304 p.

[26] Merchant, C. (1980), op.cit.

[27] Merchant C. (1981), Earthcare : Women and the environment. Environment : Science and Policy for Sustainable Development, 23(5), 6-40.

[28] Merchant, C. (1980), op.cit., 169

[29] Merchant, C. (1980), op.cit., 168

[30] Hadot P. (2004), Le Voile d'Isis Essai Sur l'Histoire de l'Idée de Nature, Paris : Gallimard, $400 \mathrm{p}$.

[31] Daston, L. \& Galison, P., (2007), Objectivity. NY : Zone books, $504 \mathrm{p}$.

[32] Pestre, D. (2013) A contre-science. Politiques et savoirs des sociétés contemporaines : Politiques et savoirs des sociétés contemporaines, Paris : Le seuil, 7.

[33] Hadot, P. (2004), op. cit., 391

[34] Horkheimer, M. \& Adorno, T.W. (1974), La dialectique de la raison : fragments philosophiques, Paris : Gallimard, 288 p.

[35] Ruphy, S. (2015), Rôle des valeurs en science : contributions de la philosophie féministe des sciences, 51, 41-54.

[36] Burgart Goutal, J. (2018). L'écoféminisme et la France : une inquiétante étrangeté ? Cités, 73, 67-80.

[37] Ingold, I. (2017) Prêter attention au commun qui vient, Conversation avec Martin Givors \& Jacopo Rasmi, Multitudes, 68, 168.

[38] Givors M.\& Rasmi J. (2017), Hors Champ, Petite introduction à la lecture de Tim Ingold, Multitudes, 68, 156.

[39] Meulemans, G. (2017), The lure of pedogenesis. An anthropological foray into making urban soils in contemporary France. Thèse en anthropologie. Univ. d'Aberdeen et univ. de Liège. [40] Macy, J. (1981), Despair work. Evolutionary Blues. 1(1), 3647.

[41] Macy, J. \& Brown, M. Y. (2008), Écopsychologie pratique et rituels pour la Terre, Gap : Le Souffle d'or, $248 \mathrm{p}$.

[42] Hache, E. (2011), op.cit.

[43] Macy, J. (1981), op. cit.

[44] Hadot, P. (2004), op. cit., 400-401.

[45] Hubert H. \& Mauss M. (1897), Essai sur la nature et la fonction du sacrifice, L'Année sociologique, 2, 29-138.

[46] d'Onofrio, S. (2014), Les fluides d'Aristote. Lait, sang et sperme dans l'Italie du Sud, Paris : Les Belles Lettres, 192 p.

[47] Tétart, G. (2004), Le sang des fleurs : une anthropologie de l'abeille et du miel, Paris : Odile Jacob, 284 p.

[48] Héritier, F. (2002), Masculin Féminin II : Dissoudre la hiérarchie, Paris : Odile Jacob, 2002, 448 p.

[49] Guattari, F. (1989), Les trois écologies, Paris : Galilée, 80 p.

[50] Blanc, N. \& Legrand, M., (2018), Vers une recherchecréation : explorer la portée transformatrice des récits dans les relations au milieu de vie, $A C M E$, (à paraître) 


\begin{tabular}{|c|c|}
\hline & $\begin{array}{l}\text { [51] Beauchêne, S. (1999), La fête du Rhône, un rite éphémère. La } \\
\text { célébration d'une identité régionale au service d'un aménagement } \\
\text { fluvial, Le monde alpin et rhodanien, 1-3, 159-174. } \\
\text { [52] Jordan, J. (2005), Faire la guerre avec amour. Vacarme, 31, 34- } \\
\text { 37. } \\
\text { [53] Starhawk (2004), Parcours d'une altermondialiste. De Seattle } \\
\text { aux Twin Towers, Paris : Empêcheurs de penser en rond, } 150 \text { p. } \\
\text { [54] Houseman, M. (2010), Des rituels contemporains de première } \\
\text { menstruation, Ethnologie française, 40, 57-66. } \\
\text { [55] Stengers, I. \& Neyrat, F. (2010), Rêver l'Obscur, c'est } \\
\text { justement défaire son opposition avec la Lumière. Multitudes, 41, } \\
\text { 176-184. } \\
\text { [56] Povinelli, E.A. (2018), Mother Earth : Public Sphere, } \\
\text { Biosphere, Colonial Sphere, E-flux, } 92 \text { (en ligne) } \\
\text { [57] Abram, D. (2013), Comment la terre s'est tue. Pour une } \\
\text { écologie des sens, Paris : La découverte, 250 p. }\end{array}$ \\
\hline $\begin{array}{l}\text { Droits des images } \\
\text { Pictures copyrights }\end{array}$ & $\begin{array}{l}\text { Droits des images } \\
\text { Figure } 1 \text { a creative commons } \\
\text { Archive } \\
\text { Figure } 1 \text { b creative commons Nickmard Khoey Historical } \\
\text { Figure } 2 \text { et suiv : @ Anaiis Tondeur }\end{array}$ \\
\hline
\end{tabular}

\section{Introduction}

Féminité. Rythme ternaire à souffler à l'oreille des fauves.

Un rebond qui valse et vole.

Une réponse clamée du haut des monts, du fond des mers

Aux forces qui nous manquent. A nos actes niés.

La terre-mère réceptacle, support inerte où pousse la graine laborantine.

La terre - femmes réceptacle, du plaisir à consommer sans frein. 
La terre. Fauve, rapace, poulpe.

Voilà qu'elle se réveille au creux de mon ventre.

Qu'elle se réveille et réclame toute mon attention.

Cet article présente une expérimentation entre art et anthropologie menée de 2015 à aujourd'hui au cours de résidences partagées ${ }^{1}$. Cette expérimentation a été développée au contact de différents publics et consiste en l'invention de rituels impliquant des offrandes de fluides corporels féminins à différents milieux de vie. Elle invite par ce biais à l'élaboration d'une éthique qui prenne appui sur la dimension physiologique de l'existence humaine - dans son versant féminin - pour prendre acte des interdépendances profondes qui nous relient avec la biosphère, comprise comme l'ensemble des êtres vivants en relation avec leurs milieux.

Ce projet s'inscrit dans la lignée d'une critique du travail conjoint de domination des femmes et de la nature, clairement dénoncé depuis une génération par le mouvement écoféministe. Le terme écoféminisme, attribué à la française Françoise d'Eaubonne [1,2] a été surtout popularisé par les luttes environnementales portées par des femmes dans les années 1980 et 1990, aux Etats-Unis mais aussi en Inde [3]. Considérant que c'est la même matrice idéologique qui structure la situation de dévalorisation et de domination dans laquelle se trouvent la nature et les femmes, les écoféministes élaborent une réflexion critique à l'égard de l'idée de nature telle qu'elle a été pensée dans la modernité ainsi que sur la façon de concevoir la féminité à cette même période.

\footnotetext{
${ }^{1}$ La première résidence s'est déroulée en 2015 - 2016 au domaine départemental de Chamarande (Essonne), au sein du projet Laboratoire de la culture durable (COAL). Le premier volet du triptyque, Galalithe, a été créé à cette occasion. Le deuxième volet, Selenhydre, a été développé de façon autonome. La résidence d'Anaïs Tondeur au Centre Tignous d'art contemporain, à Montreuil en 2017 - 2018 a permis de de travailler ensemble au troisième volet du triptyque, l'Appel aux larmes.
} 
Cette critique, ancrée dans l'action, donne lieu à un geste de réappropriation, réhabilitation et réinvention (traduction du terme anglais Reclaim) des notions de nature et de féminité. L'objectif est de déployer une nouvelle puissance d'agir conjointement sur la position subordonnée des femmes dans les sociétés contemporaines et sur les drames écologiques, les deux problèmes étant ici considérés comme intimement liés [4]. Près d'un demi-siècle plus tard, qu'est-ce qu'une approche féministe peut encore apporter à la réflexion écologique, en particulier sur le plan éthique?

Aujourd'hui, il est encore question de réparer et revendiquer ces associations entre les notions de femmes et de nature pour en faire autre chose, de manière à recouvrer un pouvoir d'agir et trouver, en tant qu'êtres humains, les termes de relations faites de respect avec la biosphère. Nous affirmons qu'il est encore nécessaire de comprendre en quoi ce travail de restauration n'est pas seulement une question technique mais aussi sociale et mentale, qui engage la subjectivité. Notre travail consiste donc à élaborer une analyse qui se place sur ce dernier plan. Plus précisément, il s'agit à partir d'élaborations à la fois matérielles, gestuelles et textuelles, de développer une pratique à même d'accompagner le déploiement de nouveaux points de vue sur le monde.

Les expériences que nous menons s'inscrivent en relation avec les travaux d'un collectif interdisciplinaire que nous avons participé à fonder, Chaoïde ${ }^{2}$. Celui-ci vise à élaborer des méthodes d'explorations hybrides entre arts, anthropologie et écologie [5]. Le premier rituel que nous avons imaginé a pris la forme d'une performance, intitulée Galalithe. Nous l'avons développée en 2015-2016 durant la résidence où nous nous sommes rencontrées, le «Laboratoire de la culture durable » co-organisée par $\mathrm{COAL}^{3}$ et le Domaine de Chamarande,

\footnotetext{
${ }^{2}$ www.chaoide.com

${ }^{3}$ L'association COAL Art et Développement Durable, a été créée en France en 2008 par des professionnels de l'art contemporain, du développement durable et de la recherche dans le but de favoriser l'émergence d'une culture de
} 
dans le lieu éponyme, aux limites de l'Essonne. Nous avons eu la chance d'être les premiers résidents avec l'écologue Alan Vergne, l'anthropologue Germain Meulemans et la designer Yesenia Thibault avec le marrainage de la géographe Nathalie Blanc. Ensemble, nous avons travaillé durant quasiment un an autour des questions ouvertes par les sols urbains et à l'intérêt de renouveler leur exploration sur un mode relationnel. C'est là qu'est né le premier rituel, consistant en une offrande de lait maternel aux êtres vivants des sols, au premier rang desquels, les lombrics. Avec cette performance, nous entamions une réflexion sur la rupture matérielle et symbolique que nous entretenons avec nos milieux de vie de façon à ouvrir des espaces de pensée et des façons d'agir qui laissent à nouveaux les autres vivants enrichir nos existences.

Il s'est rapidement imposé que Galalithe aurait une suite, deux autres rituels aussi performés à partir de nos corps et impliquant d'autres milieux de vie, d'autres éléments. La performance Selenhydre, prenant appui sur le sang menstruel, a eu lieu à l'été 2017, au cœur d'un estuaire Breton. Nous en avons rendu compte lors du colloque «La vie à l'œuvre », organisé en novembre 2017 au Musée de la Chasse et de la Nature à Paris, devant un public d'artistes et de chercheurs. Nous annoncions alors la préparation du troisième volet du triptyque, L'appel aux larmes. Ce dernier, toujours en cours de déploiement, a été marqué par un évènement public organisé en février 2018 au Centre Tignous d'Art Contemporain situé à Montreuil (Seine-SaintDenis), avec l'importante contribution de Joanne Clavel. Il rassemblait des personnes de tous âges ayant en commun de se sentir concernées par les questions environnementales. D'un rituel à l'autre, le cercle des personnes impliquées s'est ainsi progressivement élargi, accompagnant le développement de la réflexion dont cet article propose une synthèse.

l'écologie et d'accompagner la transformation des territoires par l'art. 
Après nous être positionnées vis-à-vis de l'histoire de l'éthique environnementale, nous décrirons la manière dont les trois performances du triptyque ont été produites comme expression incarnée des relations entre les existences humaines et leurs milieux de vie. Enfin nous aborderons plus en détails l'invention de rituels comme articulation entre pratique poétique et politique, à même d'asseoir une posture éthique.

\section{Contexte : déconstruire la subjectivité cartésienne}

Cette première partie propose de resituer les expériences menées au sein du triptyque dans un ensemble de courants philosophiques et éthiques qui apportent des éléments de nature à déconstruire une forme de subjectivité héritée de la révolution cartésienne. Comme l'a montré la philosophe Carolyn Merchant [6] l'essor de l'entreprise scientifique et technique qui intervient au cours de la renaissance en Europe a marqué le point d'orgue d'un mouvement de réification de la nature, à la fois réduite à de simples rouages régis par des lois immuables et entièrement cernables, et identifiée à une femme à soumettre. Cette approche du monde, aux racines anciennes, mais qui n'a jamais été universelle, offre aux mains humaines quantité de leviers et manettes, d'outils conceptuels et techniques, au moyen desquels il serait moralement loisible de manipuler le monde vivant sans limite et sans avoir à apporter de justification. Dans le même temps il serait loisible de cantonner les femmes au travail de reproduction biologique, à la partie «naturelle » de l'existence humaine, dénuée de créativité.

Le projet de déconstruction de la subjectivité cartésienne est commun, pour des raisons différentes mais connexes, à l'approche écoféministe, à plusieurs courants de l'éthique 
environnementale, ainsi qu'à certains anthropologues ${ }^{4}$. Nous inscrivant également dans cette visée, il nous apparait néanmoins essentiel de souligner que nous cherchons à conserver à notre action et à notre réflexion un ancrage matérialiste. Notre éducation s'est faite ainsi : les continuités et équivalences que nous pouvons reconnaitre entre les différentes formes d'existence qui peuplent la terre sont d'abord liées à la matière. Et c'est en reprenant appui sur cette base que nous tâchons d'aller vers une forme d'extériorité réflexive visant à proposer d'autres formes relationnelles, alternatives à la réification et l'exploitation pure.

\subsection{Préoccupations morales pour l'environnement}

L'empreinte toujours croissante des activités humaines sur les milieux de vie a conduit, au cours de l'histoire récente, des acteurs de tout ordre à chercher à développer de nouvelles éthiques vis-à-vis de la biosphère, qui passent tant par l'élaboration théorique que par l'expérimentation. Du point de vue philosophique, les différents courants émergés au cours du vingtième siècle diffèrent fortement dans leurs fondements comme dans leurs conséquences pratiques.

Un balancement a longtemps prévalu entre deux conceptions opposées de la protection de la nature, pour des raisons utilitaristes comme pourvoyeuse de biens et services ou pour des raisons esthétiques et spirituelles comme porteuse d'une valeur intrinsèque [9]. Les positions des institutions internationales ont longtemps circulé en un va et vient constant entre ces deux tendances [10]. L'avènement de la notion de biodiversité participe à un dépassement de cette opposition.

\footnotetext{
${ }^{4}$ Du côté de l'éthique environnementale, c'est surtout vrai pour le courant éco-centriste, qui invite à se mettre en situation de penser pour tout un écosystème [7]. Du côté de l'anthropologie, on trouvera un exemple clé avec la proposition perspectiviste menée par Eduardo Viveiros de Castro [8], qui prend appui sur des modes de pensée liés à l'animisme sud-américain pour mener une entreprise de décolonisation de la pensée.
} 
En premier lieu, l'évolution du rapport des sciences à la notion d'équilibre a influencé les tenants d'une valeur intrinsèque de la nature. Différentes théories de la valeur intrinsèque des éléments naturels émergent, avec en particulier les travaux d'Aldo Leopold et Baird Callicott, qui mettent pour ce faire en relation les concepts moraux et biologiques de parenté et de communauté [11]. Leopold s'appuie fortement sur le paradigme odumien de l'équilibre écologique pour développer ses travaux sur l'intégrité écologique [12]. Callicott par la suite prend acte de la remise en cause de la dualité cartésienne entre sujet et objet et entre fait et valeur qui découle, notamment, du développement de la théorie quantique [13] et renouvelle l'articulation entre questions éthiques et rationalité scientifique [14]. Il s'appuie sur une définition de la nature comme ensemble dynamique duquel participent toutes les productions humaines, au sein d'une hiérarchie de systèmes reliés entre eux par des propriétés émergentes, s'exprimant au travers de la diversité des formes de vie, et pouvant être mis en péril dans son fonctionnement par l'expansion de la technique [15].

En second lieu, prendre acte de la transformation permanente du monde vivant pour construire un système de valeurs conduit au développement d'une éthique évolutionniste : ainsi, selon les fondateurs de la biologie de la conservation, les humains se sont rendus responsables de l'évolution [16]. La diversité biologique devient un héritage, essentiel à entretenir dans la mesure où la présence de la vie sur Terre tient à la possibilité qu'elle a de changer. Il s'agit donc de conserver les possibilités de transformation, et non plus l'intégrité écologique.

Un ensemble de propositions pragmatiques s'est également développé, de façon plus récente. Retenons seulement ici la proposition « cosmopolitique » d'Emilie Hache qui, sur les traces d'Isabelle Stengers, invite à élargir la communauté morale afin de prendre en compte un plus grand nombre d'existants dans nos décisions. Il s'agit ainsi comme elle le propose de «prendre soin de ce cosmos en veillant à ce que ce à quoi les gens tiennent ne soit pas oublié ou maltraité - en collectant ces demandes morales et en les faisant exister, en contribuant à y intéresser des 
protagonistes hétérogènes » [17]. C'est à une démarche pragmatique et attentive à la diversité culturelle que ces discours invitent.

Ces différents courants, à l'exception du dernier, ont en commun de se situer d'abord sur un plan logique, reliant argumentation réflexive et considérations morales. Le présent travail relie, lui, l'élaboration éthique à un argumentaire situé sur le plan des relations entre subjectivité, sensibilité et attention. Il s'ancre, pour ce faire, dans une reprise critique de l'histoire des assimilations entre les femmes et la nature, comme objet de connaissance et d'exploitation, telle que déployée par le mouvement écoféministe.

\subsection{Mettre fin à l'éthique de l'exploitation}

L'avènement de la modernité, qui associe révolution scientifique, multiplication des machines et essor de l'extractivisme, s'accompagne d'un véritable renversement de la place des figures féminines invoquées pour symboliser les rapports productifs à la terre.

Précisons que la modernité est entendue ici, non seulement comme une période historique, mais surtout comme accentuation permanente d'un rapport volontariste à l'environnement, fait d'interventions directes positives, de domestication, d'artificialisation et de tentatives de plus en plus efficaces de maximiser la productivité immédiate. Cette conception, commune aux visions capitalistes et marxistes des systèmes de production, forme une assise pour la transformation toujours accélérée des ressources naturelles en valeur économique grâce au travail et au progrès technique, tous deux sacralisés ${ }^{5}[18,19]$.

\footnotetext{
${ }^{5}$ La question de la conscience environnementale de Marx a été débattue dès les années 1930 [22] et le marxisme forme aujourd'hui une assise à des courants anti-productivistes tels que l'écosocialisme [23]. Par ailleurs, comme le souligne Jean-Baptiste Fressoz [24], les philosophes modernistes ne dissociaient pas tous complètement histoire humaine et histoire de la nature, en particulier lorsqu'ils considéraient les questions métaboliques et climatiques. Marx est de ceux-là, dans son analyse des ruptures métaboliques entre ville et campagne.
} 
Ainsi l'effacement moderne des figures féminines de la fécondité comme puissance, s'inscrit dans une transformation plus large des imaginaires, concomitante d'une transformation des rapports de production et plus largement des rapports sociaux. La venue de la modernité, comme le montre Castoriadis [20] s'accompagne d'un double mouvement de ce qu'il nomme l'imaginaire social : d'une part comme projet d'émancipation individuelle et collective, et d'autre part, comme désir de maitrise rationnelle de la nature. Or ce dernier passe en particulier par le développement des sciences comme rapport privilégié à la nature, faite d'acquisition de connaissances conçues comme un dévoilement. Dans ce mouvement, l'image de la terre-mère, dont la puissance s'était déjà progressivement affaiblie dans les époques précédentes, disparait alors véritablement pour laisser la place à celle d'une terre inerte rendue analogue à une femme à soumettre sexuellement. Cette entité inerte se trouve alors considérée comme objet d'exploration intellectuelle et d'exploitation technique.

Cette transformation s'inscrit dans le temps long. Françoise d'Eaubonne [21] montre ainsi comment le déploiement historique du patriarcat, comme accaparement masculin de la capacité reproductrice de la terre et des femmes, et ce depuis le néolithique, est allé de pair avec un effacement progressif de la sacralité féminine. Si nous cherchons à remonter au plus loin que nous pouvons le fil de l'émergence de ce processus, il apparait que trois révolutions religieuses - et donc symboliques, ont rendu possibles les transformations techniques et politiques des rapports à la production, en particulier alimentaire [25]. La première étape concerne la révolution du néolithique. Datée entre -10000 et -9000 , elle voit l'apparition de trois types de cultes combinés : divinité féminine, divinité taurine et culte des crânes et des morts, ce dernier formant les prémices d'un culte de l'homme lui-même. Cette divinité féminine est associée avec une grande continuité jusqu'au $2^{\text {ème }}$ siècle après JC à une multiplicité de seins et à des lions, symbolisant l'ambivalence du rapport humain à la terre, entre nourricière et menaçante. Artémis d'Ephèse est sa dernière représentation connue (figure 1a). La deuxième étape est 
marquée par une séparation entre les deux faces de la divinité féminine dans le panthéon. Fertilité et puissance menaçante sont distribuées à différentes figures, Déméter et Athéna par exemple représentant la mère et la guerrière. La troisième étape se trouve dans la religion chrétienne, qui prenant son ampleur amène enfin un dieu masculin et sans chair dans le ciel, tandis que la vierge s'efface largement : la divinité féminine disparait pour prendre le rôle de véhicule de la filiation masculine de Dieu.

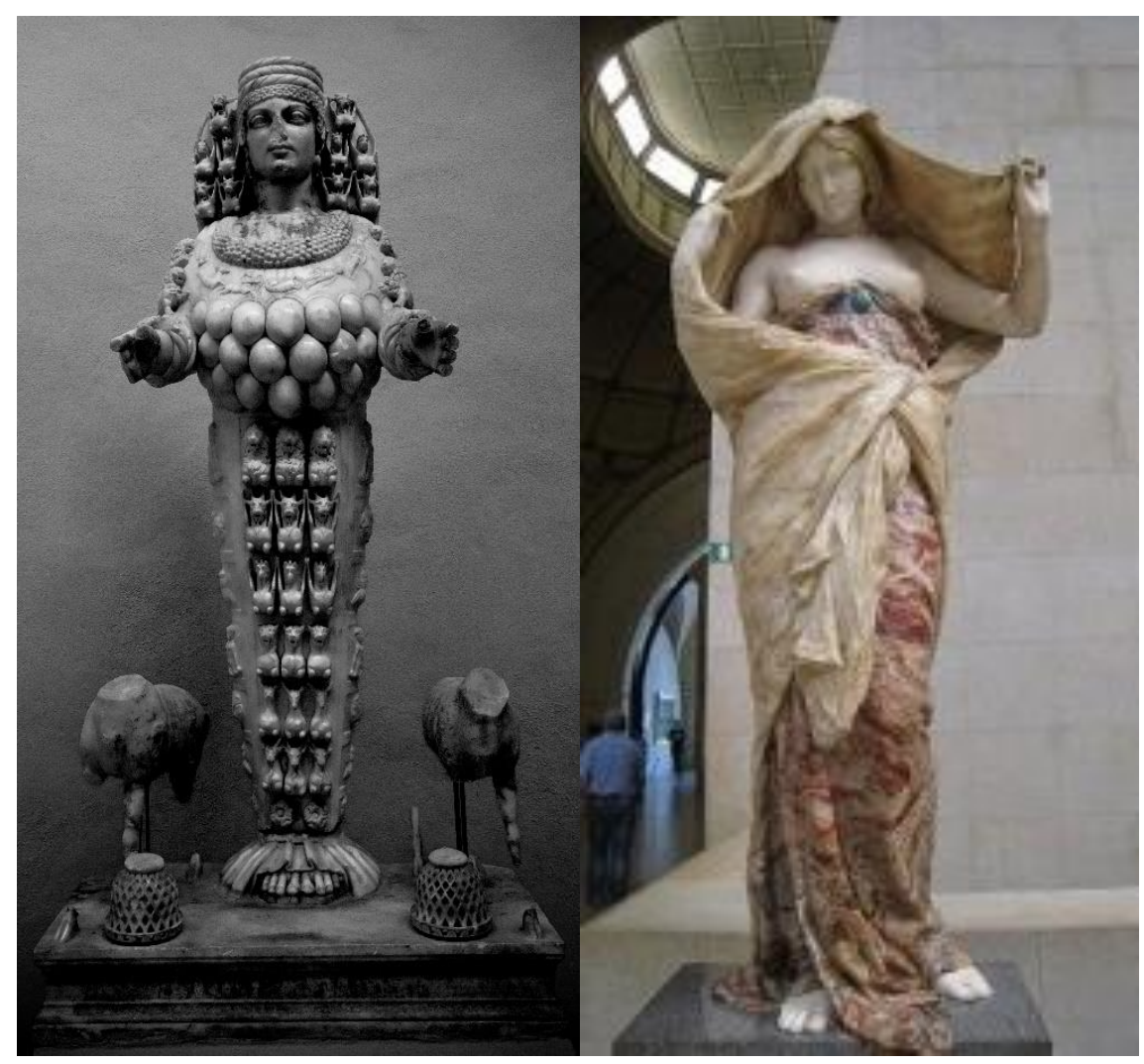

Figure 1. a : statue représentant Artémis d'Ephèse (cc) Nickmard Khoey Historical Archive; b. statue intitulée « La nature se dévoilant devant la science » Ernest Barrias. (cc) Daniel Smullen

Une étape de plus est franchie vers une domination de plus en plus totalitaire de la nature identifiée à une figure féminine. Nous faisons ici en particulier référence au travail de Carolyn Merchant [26, 27] et à l'interprétation qu'elle propose des textes de Francis Bacon comme signe 
de l'avènement d'une nouvelle éthique de l'exploitation. Au début du XVII ${ }^{\text {̀̀me }}$ siècle, dans The Masculine of Birth Time celui-ci affirme : «je suis venu en vérité, menant à vous la nature avec tous ses enfants pour la mettre à votre service et faire d'elle votre esclave (...) nous n'avons pas le droit d'attendre que la nature vienne à nous...la nature doit être attrapée par le toupet, chauve qu'elle est à l'arrière » (cité par Merchant [28]). Les techniques nouvelles, les arts mécaniques qui prennent à l'époque où il écrit un essor énorme permettront enfin d' "envisager les secrets toujours enfouis dans le giron de la terre » (ibid.). L'artificialisation via l'usage d'une approche mécaniste est clairement explicitée comme un projet de domination qui s'appuie sur une « imagerie sexuelle brutale » que Merchant considère comme « la clé de la méthode expérimentale moderne - la contrainte de la nature en laboratoire, la dissection manuelle et mentale, et la pénétration des secrets cachés » [29]. L’articulation de la destruction de la nature et de l'oppression des femmes ressemble à un ruban de Möbius : les femmes sont inférieures parce qu'elles font partie de la nature, et on peut maltraiter la nature parce qu'elle est féminine. Francis Bacon, s'il reste dans les mémoires comme le plus violent, n'est pas le seul à employer ces analogies. Ainsi, la représentation en statuaire de la nature comme une femme au corps en voie de s'offrir est récurrente jusqu'au XIXème siècle [30].

\subsection{De la nature à dévoiler au retour de la phusis comme émergence}

La quête scientifique s'assimile depuis la Renaissance à une tentative de dévoilement des secrets de la nature, trésors qu'elle garderait jalousement et qu'il faudrait lui arracher. Mais qu'entend-on exactement par dévoilement ? La science est considérée par les savants comme la recherche d'une vérité au-dessus des opinions. Elle est désir d'objectivité et pourtant, elle est prise dans un jeu de mise en scène $[31,32]$. Pour reprendre l'image précédente, La Nature se dévoilant devant la Science, dans la version du XIXème siècle ${ }^{6}$, représente une femme qui

\footnotetext{
${ }^{6}$ Statue en marbre polychrome produite par Ernest Barrias en 1899, elle est exposée encore aujourd'hui au Musée d'Orsay à Paris. Elle a fait l'objet à l'époque de nombreuses reproductions et gravures.
} 
soulève son voile pour se dénuder jusqu'à montrer ses seins en gardant une posture modeste, tête baissée (figure 1.b). Qui est-elle? Il s'agit, ce n'est pas une coïncidence, d'une représentation d'Isis. Or les figures d'Isis et Artémis, dont nous parlions plus haut, ont régulièrement été assimilées, et ont largement circulé ensemble dans l'histoire de la pensée européenne.

La figure antique d'Isis sert ainsi de point de référence récurrent pour évoquer l'entreprise scientifique de dévoilement. Un aphorisme, «la nature aime à se voiler », que l'on doit à Héraclite, sera en particulier continuellement discuté au cours des siècles, en lui donnant selon les périodes des significations différentes, traduisant les changements de regards sur les relations entre nature et connaissance. Cet aphorisme a en effet pu aussi bien justifier l'attitude prométhéenne concomitante du déploiement des sciences modernes, que l'attitude orphique notamment représentée par le mouvement romantique, au XIX ${ }^{\text {ème }}$ siècle, faite d'effroi, d'extase, et d'une exigence du respect des mystères naturels.

Or au vingtième siècle, plusieurs philosophes, tels qu'Heidegger, Merleau-Ponty ou encore Wittgenstein, proposent des voies différentes pour développer une relation au monde qui ne soit pas ancrée dans le dévoilement. Il faut noter qu'il n'est alors même plus question de nature mais de l'Etre, en général. Un retour est alors pourtant opéré sur le sens originel du terme grec phusis. Ainsi pour Heidegger, «l'idée occidentale de nature, dans ses origines, résulte de la vision grecque de l'Etre comme éclosion, comme émergence » et celui-ci comprend donc la phusis d'Héraclite dans le sens d'éclosion, action de faire apparaitre [33].

Selon cette conception, Isis, ancienne incarnation de la nature comme cible de la quête scientifique, serait donc inséparable de ses voiles. Il ne s'agit pas de lui enlever, personne n'a à lui enlever, puisqu'elle se manifeste, de façon non séparée de l'existence humaine, en tant que phusis, par un acte de dévoilement actif. La figure figée qui reprend vie quitte le statut d'objet 
que l'on dévoile pour acquérir celui de puissance d'agir, de dynamique de constant déploiement. Cette ouverture est inséparable d'un voilement, d'une part de secret, d'obscur, toujours conservé. Ce dernier renvoie tel que nous le comprenons, à ce qui est, en puissance, c'est-à-dire en potentiel, source et ressort. Et donc également, à la puissance d'agir.

\subsection{Eduquer notre attention}

Notre proposition s'inscrit en réponse à la violence de la réification qui va avec toute objectivation. Celle-ci a été dénoncée par Adorno et Horkheimer [34] dans leur critique de la rationalité instrumentale. Cette critique a été approfondie par le mouvement féministe, qui l'a enrichie, en particulier, d'une attention aux asymétries de genre dans la pratique scientifique $[35]^{7}$.

Pour notre part, nous situons notre travail au cœur des relations entre corps, langage et environnement, pour retrouver une forme d'attention qui se serait évanouie des relations quotidiennes. Nous entendons ici les relations entretenues avec l'autre, avec l'environnement en général et donc en particulier, avec les autres êtres vivants et la matière qui les constitue et transite à travers eux. Cette recherche n'est pas tournée vers une quelconque révolution individuelle des consciences. Elle est collective, politique. Sur ces bases, notre proposition s'appuie sur l'attention, au double sens du terme, sensible et éthique. Cette attention au monde prend racine dans la sensibilité au sens de perception directe et non instrumentée de l'environnement, comme l'explique l'anthropologue Tim Ingold. Celui-ci évoque ainsi une «insensibilité fondamentale» liée au développement d'un «corps armure » associé à

\footnotetext{
${ }^{7}$ Notons que pourtant et paradoxalement, ce mouvement reste imprégné en France du dualisme qui marque fortement celle est aujourd'hui considérée comme sa fondatrice, Simone de Beauvoir. Ainsi, les conceptions écoféministes y font une percée beaucoup plus lente qu'ailleurs dans le monde [36].
} 
l'hypermédiatisation des relations avec l'environnement. Cette insensibilité se révèle selon lui problématique pour aller contre les désastres écologiques :

(...) globalement, nous avons perdu une sensibilité immédiate à notre environnement - «nous » désignant ici les gens en général, ceux qui ne font plus attention à ce qui les entoure, nous devions et étions habitués à le faire auparavant. Chez beaucoup de personnes, la technologie a généré une forme d'immunisation, une sorte de corps-armure qui nous empêche d'avoir à négocier directement avec l'environnement [37]

Allant plus loin, il s'agit d'éduquer à nouveau l'attention en se défaisant d'une volonté de maitrise, décrite par Yves Citton comme également partie prenante d'un détachement du réel [38]. Il propose pour ce faire (en référence à Jan Masschelein) d'adopter une approche de l'attention qui renvoie non seulement à l'action de percevoir le monde, mais aussi à celle de comprendre quelles sont les intentions et les besoins qui s'y expriment, de façon à y répondre :

(...) attendre, en français, signifie attendre que quelque chose arrive [to wait], et donc que faire attention à quelque chose [to attend to something] n'est en rien une maitrise, mais bien plutôt une forme de soumission. Cela consiste à attendre le monde [waiting upon the world], à être à la fois attentif aux choses et attentionné envers les personnes, selon le modèle du serveur [waiter] dans un restaurant, qui fait attention à ses clients et se tient prêt à faire ce qu'ils lui demandent : le serveur est du côté du service plutôt que de la maîtrise (ibid).

Voici donc ce que nous cherchons à faire : retrouver une attention aux liens qui nous unissent aux milieux de vie que nous habitons et des capacités empathiques envers des êtres nonhumains. Là où les sciences ont longtemps procédé par dévoilement pour donner accès à ce que les sens ne perçoivent pas, par le biais d'instruments, les pratiques que nous mettons en place, à la jonction entre texte, geste et travail de la matière, offrent un autre accès à la connaissance ce qui ne se sent pas ordinairement, en tout cas dans le contexte où nous avons grandi : la fluidité des existences, les profondes continuités entre les corporéités humaines, leurs milieux, largement peuplés d'autres êtres vivants qui mènent aussi leur vie chacun à leur manière : ce que sans cesse, nous partageons et échangeons. 


\section{Manipuler les fluides corporels féminins : une expérience entre art et anthropologie}

Depuis quatre ans, nous avons entrepris, au travers d'un cycle de performances de faire subir une manipulation particulière à plusieurs fluides corporels féminins, ou considérés comme tels : le lait, le sang et les larmes. L'expérience que nous menons vise à développer des figures relationnelles qui permettent d'appréhender l'enchevêtrement entre les existences humaines, dans toute leur épaisseur, avec l'ensemble de la biosphère. Ces performances cherchent à produire des images à même d'accompagner un processus de subjectivation. Elles se construisent dans un mouvement de détachement vis-à-vis des notions modernes de nature et de femme en tant qu'entités statiques, immuables, passives, pour repenser les relations entre les existences humaines et la biosphère à partir d'un regard renouvelé sur ce qui circule, matériellement, entre les êtres vivants et les milieux de vie.

\subsection{Galalithe}

La première performance à laquelle nous avons entrepris de donner forme s'adresse aux sols. Négligés, les sols et encore plus les sols urbains sont restés dans l'ombre des sciences durant plus de 150 ans. Les sols urbains sont couramment perçus comme simple support pour la marche, matériau inerte, « boite noire technique » de la ville où circulent divers flux - eau, gaz, électricité, information [39]. Or c'est un système complexe de relations qui lie les humains aux sols et jusqu'au cœur des villes. C'est ce que nous avons tâché de mettre en mouvement au travers de cette résidence qui a abouti à l'exposition « Sols Fiction » au domaine départemental de Chamarande, au printemps 2016, et à l'organisation de plusieurs ateliers avec les publics du parc. Au-delà de révéler les relations existantes, l'idée qui nous portait était aussi de les enrichir 
et d'en proposer de nouvelles. Notre performance Galalithe, qui prend la forme de l'invention d'un rituel, en témoigne.

Le rituel prend pour support le lait maternel. Cette recherche est concomitante de la naissance de la fille d'une de nous deux. La maternité est une période où la notion d'attention, au sens de prendre soin d'un autre être, prend tout son sens. Comment transférer cette même qualité d'attention aux êtres autres qu'humains ? Nous avons choisi de nous adresser aux lombrics, organismes essentiels à la formation des sols à partir de la décomposition de la matière organique, représentants éminents des détritivores. Nous avons réalisé une large coupe à partir de concrétion de lait maternel puis déposé celle-ci en offrande aux lombrics qui habitent et transforment un sol marqué par l'histoire industrielle des marges de Paris.

Le lait maternel collecté a été transformé en une matière connue sous le nom de Galalithe, du grec gala (lait) et lithos (pierre), soit «pierre de lait». La production de la coupe suit un processus spécifique : nous avons extrait la caséine du lait qui s'est ensuite durcie lentement à la chaleur dans la forme d'une coupe d'une matière proche de l'ivoire, transparente, fragile et dure à la fois. Une fois déposée, sous l'effet des aléas météorologiques, la coupe s'est délitée. Poussière de lait, mêlée aux matières végétales, elle a participé à la pâture des vers. Les lombrics se sont emparés de cette matière jusque dans leurs galeries, l'ont digérée peut-être pour former de nouveaux sols (figure 2.a et b). 


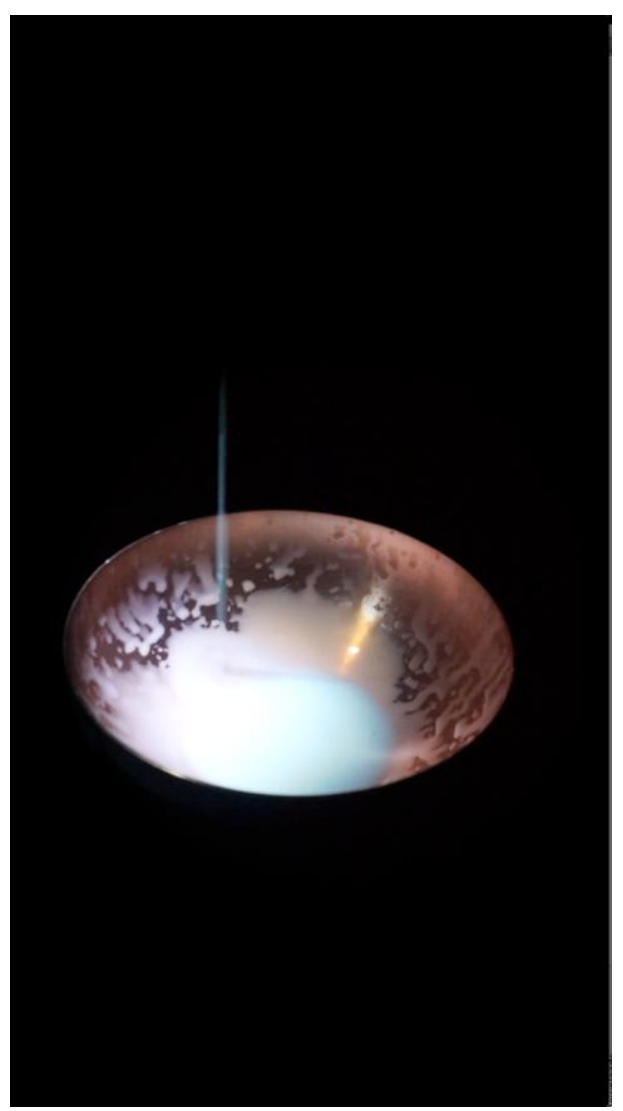

Figure 2.a. : Galalithe, Vue de la vidéo, HDV, Col, 4'57'’ 2016 (C) Anaïs Tondeur

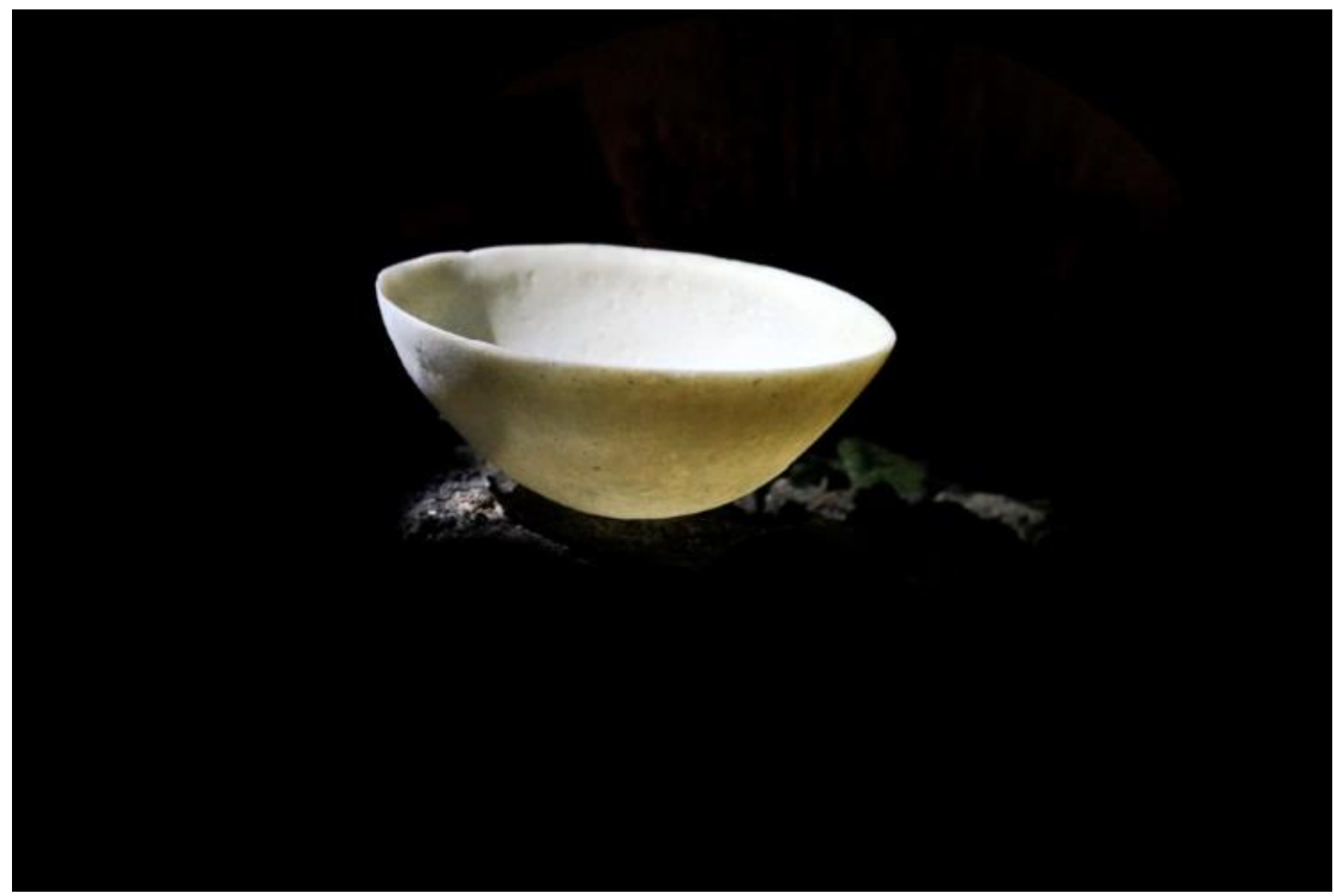

Figure 2.b : Galalithe, Concrétions de lait humain, Vue de la coupe, 2016 @ Anaïs Tondeur 
Par ce geste symbolique, cette performance participe ainsi à rendre les sols à nouveau fertiles en affirmant le lien nourricier réciproque qui nous unit au sol, support de nos vies humaines. Galalithe fait également écho au processus de digestion des vers de terre, qui assemble les particules de roches et de végétaux ingérés pour générer le véritable sol. De l'intérieur vers l'extérieur, de notre corps et à celui de l'animal, nous franchissons également avec ce travail, qui est aussi une narration particulière, la frontière entre analyse et invention, entre science et fiction. Le principe est ici de produire un geste qui nous offre en retour des ressources analytiques pour interroger nos relations aux sols.

Une trace vidéo du processus de création, d'offrande et de délitement a subsisté à la performance donnant lieu à une installation vidéo constituée d'une projection vidéo et d'une coupe réalisée en Galalithe. Il en va de même pour les deux autres rituels. Une mémoire du geste est conservée par un médium vidéo et un double de l'offrande.

\subsection{Selenhydre}

Le deuxième geste du triptyque qui prend appui dans le corps féminin pour soigner nos relations aux milieux de vie s'adresse, lui, à l'eau. Il s'agit d'une offrande de sang menstruel à l'eau d'une rivière. Ainsi, en parallèle avec Galalithe, la pierre de lait, il se nomme Selenhydre, du grec sélène (la lune) et hydr (aquatique) : il s'agit d'eau de lune.

Celui-ci émerge de l'importance, identifiée suite à la réalisation de Galalithe, de ne pas focaliser notre exploration de la féminité sur la question de la maternité. Selendydre forme donc un pendant au premier rituel, tourné vers une part plus obscure de la féminité, marquée d'un tabou profond. 
Ce deuxième geste répare un double enfermement. Celui que forme les barrages, sur les rivières, et analogue à lui, le silence qui entoure le sujet des règles, comme tare féminine voire comme malédiction. Ainsi, Selenhydre accompagne l'écoulement de l'eau entre les berges des rivières et la porosité des matières au travers desquelles elle s'accumule pour rejaillir plus loin, et tendre peu à peu vers l'océan, où sa force déferle en retour vers les terres émergées. Là où se trouve son centre. De gravité ou d'accumulation. L'océan lieu originel primordial premier plus grand plus profond. Là d'où cela jaillit. Voici, alors, le lieu ...l'embouchure d'une rivière, quelque part en Bretagne.

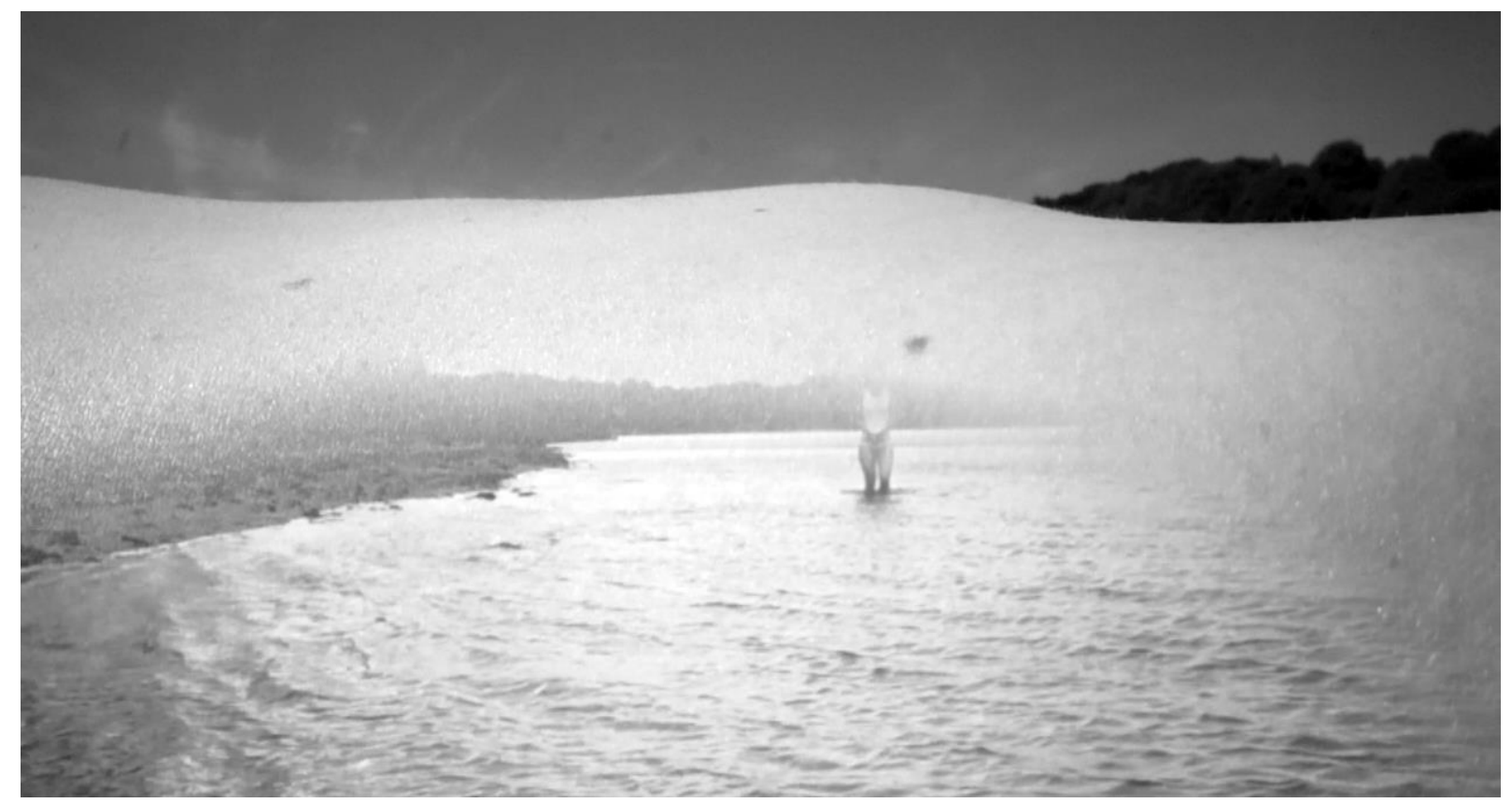

Figure 3 : Selenhydre, Vue de la vidéo, HDV Col, 5’26”, 2017 @ Anaïs Tondeur

La performance a pris la forme d'une offrande, réalisée par l'une de nous au moment du début de son cycle, dans l'embouchure d'une rivière, à marée montante (figure 3). L'expérience vécue lors du rituel et les réflexions qui en ont découlé ont été consignées en style libre. Nous reproduisons ci-dessous un extrait du texte qui en a résulté. Celui-ci a ensuite été partagé à voix 
haute, en public, accompagnée d'une vidéo produite à partir d'une prise de vue de la scène, dans l'intention de rendre compte et partager avec l'auditoire la transformation, à la fois émotionnelle et intellectuelle, opérée par la performance.

Si le sang va à l'eau, pourquoi la rejoint-il? Elle a d'abord entendu un cri. De rage. Un déchirement. Elle a marché longtemps à travers l'entremêlement des courants. Elle a remonté l'estran, avec dans ses mains la trace fantomatique d'une coupe formée de son sang et de la vase recueillie sous ses pieds qui s'enfoncent. Sur un petit amas rocheux, elle a déposé cette éloge à la marée, cette offrande comme un accompagnement de la vasière, de l'estran, du marécage sableux: ces sols imprégnés d'eaux aux formes changeantes, incertaines, lieux "riches" de vies du fait même de la décomposition dont ils sont le siège. Avec la marée montante, la coupe s'est dissoute aux eaux multiples de l'estran et de l'océan. Et celle qui marche se trouve ainsi en pensée dissoute par la puissance de l'eau ... ainsi ce liquide improprement qualifié d'impur se répand en minuscules molécules et avec lui sa soi-disant souillure s'en trouve annulée du même coup par le mélange, la dissolution réparatrice.

La marée cycle de submersion. L'eau libre qui imprègne. Lieu incertain, frange et bord, quand l'une traverse l'autre. Là où les fluides se mêlent... la vase, ce mélange de matière organique en décomposition, d'argiles et d'eau, riche et visqueuse, informe. Charriés par le cours d'eau jusqu'à sa dernière ouverture. Quand la route est barrée, les sédiments s'entassent, restes de l'érosion des parois rocheuses et des morts de l'amont. Le cours d'eau canalisé séparé de ses berges, empêché de porter, les cufs des poissons, grenouilles, moules, écrevisses, larves innombrables. Stérilité. Mais, nous répondra-t-on, la vase n'est que pourriture mauvaise et terre stérile justement, en outre impraticable, il faut l'assainir! L'assécher! Nous savons maintenant que vous vous étiez trompés. Nous revenons sur vos pas. Il faut un éloge de 
l'humidité des milieux comme cette couleur féminine, long sujet de mépris. Il faut lui laisser tout l'espace dont elle a besoin.

Faire en public le parallèle entre nos propres règles et l'écoulement de l'eau et de la vase des rivières, en remaniant le jeu d'analogie utilisé pour dévaloriser le corps féminin et les milieux marécageux a comme objectif de faire l'expérience d'une inversion qui les met en avant, l'un par l'autre.

Enfin, sur le plan matériel, de même que pour Galalithe, Selenhydre donne lieu à un double de l'offrande. Nous avons ainsi expérimenté différents processus permettant d'exposer une coupe produite à partir de sang mêlé de sable d'estuaire. Par la cuisson, du verre est produit, dans lequel quelque chose du sang est conservé intact : un élément métallique bien connu, le fer. Fer guerrier, fer ménager : ce composant essentiel du sang amène à un nouveau jeu d'analogie, qui permettra de poursuivre l'exploration plus loin. Chaque nouveau geste du triptyque ouvre donc une porte vers la trajectoire suivante, dont nous ne connaissons pas à l'avance l'issue.

\subsection{Appel aux larmes}

Dans un mouvement qui s'élargit par rapport aux deux premiers rituels, le troisième geste du triptyque propose de donner forme à un sentiment largement partagé par nos contemporains : le désespoir écologique, tel que formulé par J. Macy [40, 41], à savoir le malaise profond, la tristesse, la colère et la honte que chacun peut ressentir devant les destructions qui touchent actuellement l'ensemble des milieux de vie. Emilie Hache [42], de son côté, évoque le 
« sentiment du tragique » face aux conséquences environnementales désastreuses d'un mode de vie collectif qui déborde amplement des limites de ce que ces milieux peuvent supporter.

Ainsi, les causes de détresse en se multipliant menacent de nous faire sombrer dans un abîme d'impuissance. Pour le troisième et dernier épisode du triptyque, consacré aux larmes du deuil, le processus entamé en duo s'ouvre à un large groupe de participants. Notre horizon est ici de donner une forme au désespoir dans un geste collectif et multiple. Nous souhaitons rassembler les désespoirs que nous sommes nombreux à ressentir, rassembler nos larmes pour rendre compte de ce processus d'assèchement mortifère. Pleurer pour le monde vivant qui se disloque, se désagrège sous nos yeux. Echapper au déni. Pourquoi les larmes ? La tristesse que nous touchons va au-delà de la mort individuelle et de l'angoisse qu'elle génère. C'est la répression de l'expression de souffrance qui est celle d'un deuil pour un monde dont nous faisons partie, en train de s'affaiblir, souffrir, mourir.

L'objectif est de laisser la place à l'expression de désespoir comme moyen de retrouver un pouvoir, en envisageant le pouvoir d'une autre manière, en synergie, comme une ouverture, en se rendant vulnérable, sensible, capable de changement. Il tendra à ouvrir un espace au « travail du désespoir » [43] : s'autoriser à exprimer la peine pour un monde commun impliquant l'ensemble des vivants conçus comme interconnectés. Laisser place à la douleur pour pouvoir ensuite les sublimer en puissance pour agir en retour. Ici, le jeu de mot sur le terme sublimer est volontaire : ce verbe désigne également le passage direct de la phase solide à la phase gazeuse, sans passer par la phase liquide.

Pour donner forme à ce troisième rituel, nous avons demandé aux participants un don d'une larme. Nous les avons rassemblées dans une large coupe dans laquelle nous avons baigné les pieds du public de la performance qui prit place au Centre Tignous d'Art Contemporain, à Montreuil, en février 2018 (figure 4). Puis, nous avons laissé l'eau des larmes s'évaporer. Un 
agrégat de sel au fond de la vasque s'est formé puis s'est cristallisé donnant corps à un large disque. Le troisième geste de ce rituel évoluera de cette concrétion de larmes.

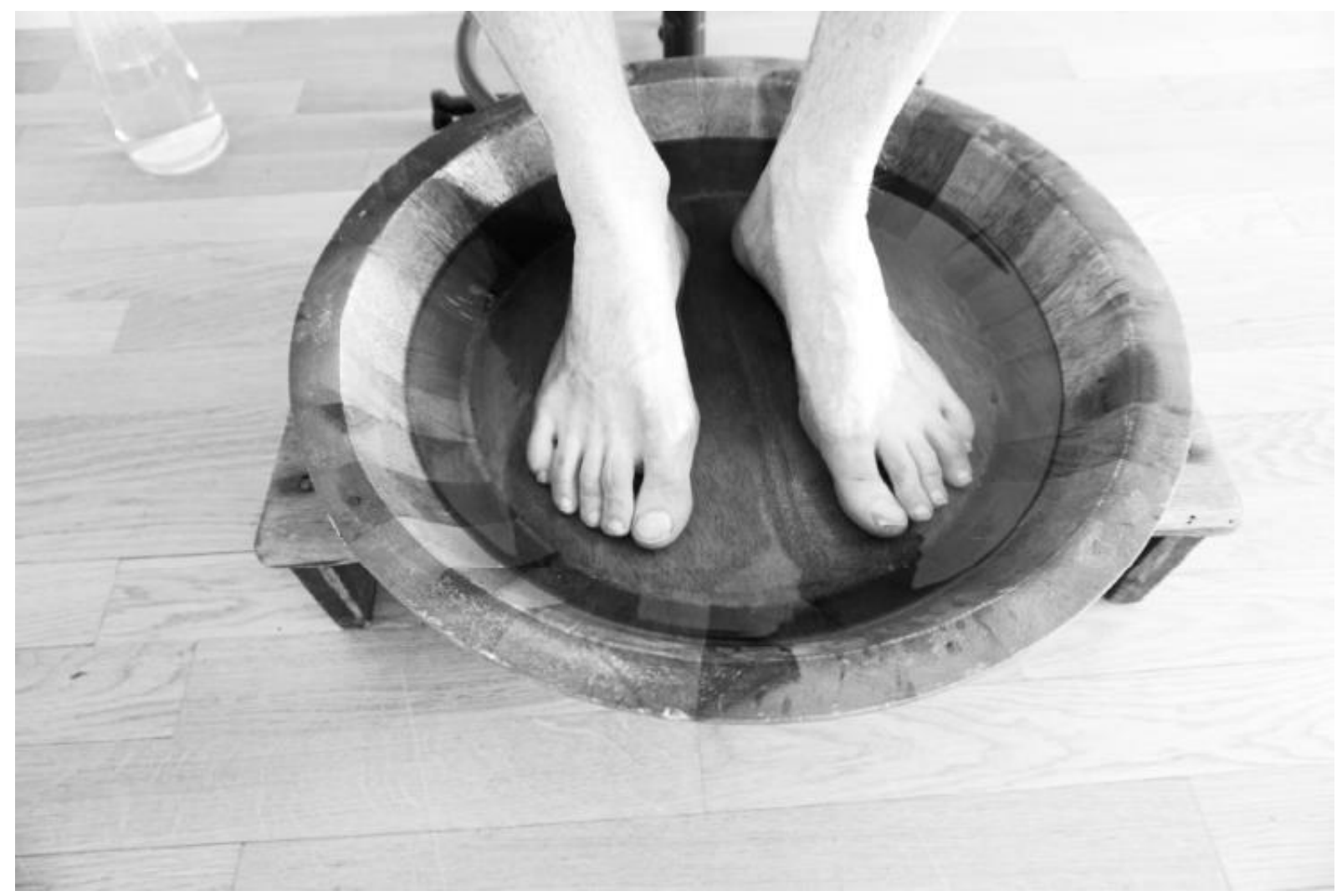

Figure 4 : Accueil des participants. Performance Appel aux Larmes, Centre Tignous d'art contemporain, Montreuil 2018 @ Anaïs Tondeur 


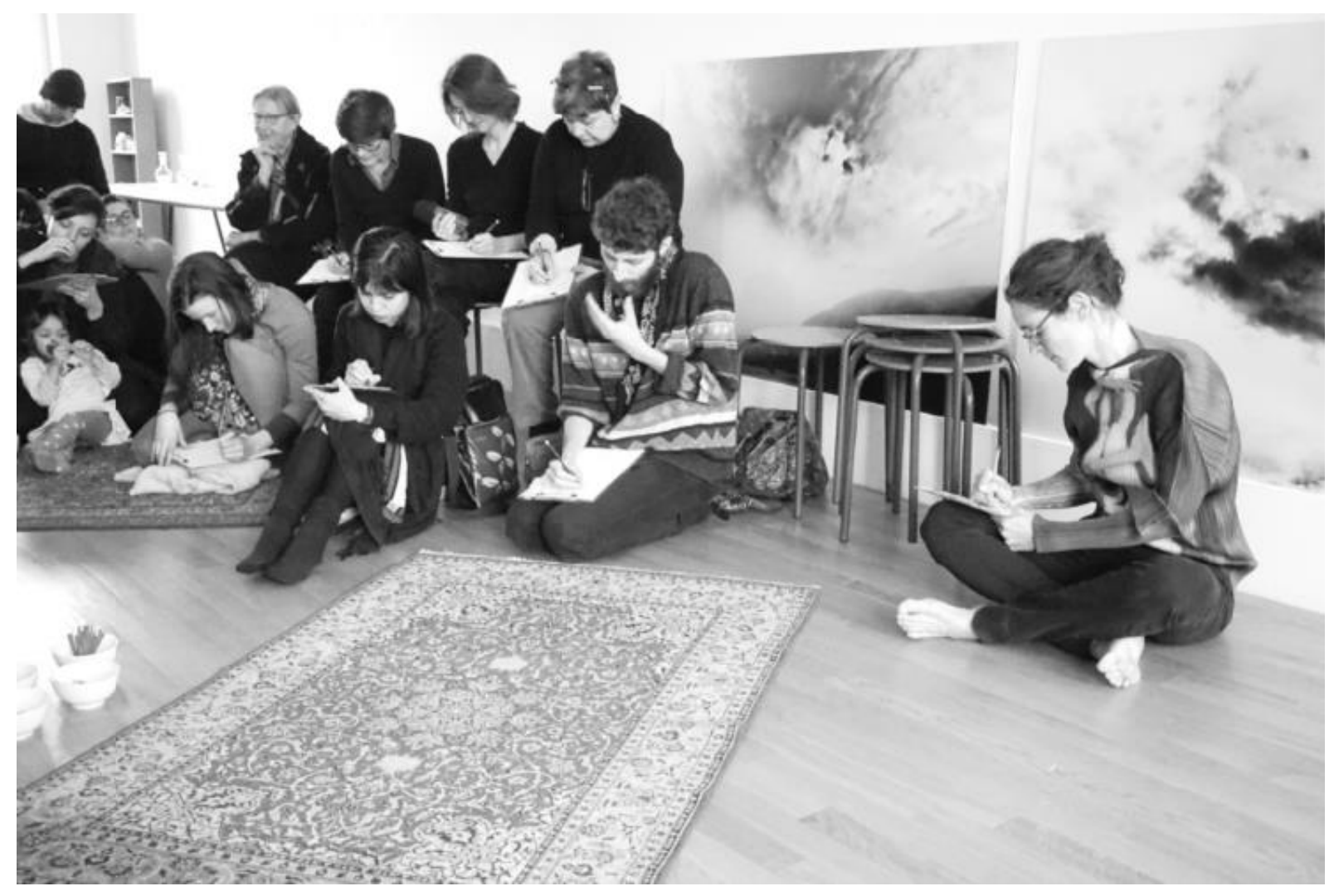

Figure 5 : Assembler nos désespoirs Performance Appel aux Larmes, Centre Tignous d'art contemporain, Montreuil 2018 ㄷ Anaïs Tondeur

Durant la performance qui préparait au dernier temps du triptyque, nous avons tenté de donner une forme collective à cette tristesse et cette angoisse mêlées. Avec l'ensemble des participants, nous les avons cartographiées à l'échelle de notre quotidien par de courts textes (figure 5). Cela a donné lieu à une litanie de mots et de couleurs que nous avons ensuite adaptée, avec un noyau de participants formés à la suite de la performance dans une composition de sons et de voix. Cette dernière reprend un grand air national et militaire au féminin et comporte plusieurs couplets. Son refrain s'entonne comme suit :

Aux larmes qui voudra bien

Arrachez vos baillons

Fermons les yeux 
Ouvrons les vannes

Coule un sang d'encre

Pour la terre en haillons

Cette composition est structurée comme une base qui pourra évoluer à l'infini. Une trame dont d'autres seront invités à s'emparer, pour la reprendre en partant d'eux-mêmes. La dernière étape n'est pas encore réalisée. Portés par un fragment de ce chant en continuelle formation, dans un large mouvement de voix et de corps, nous déposerons une nouvelle cristallisation de nos larmes dans « de hauts lieux de pouvoirs », tel qu'annoncé lors de la rencontre de février. Ces lieux peuvent être définis de différentes manières, mais c'est bien de pouvoir politique dont il s'agit. Une fois la concrétion de larme déposée, un second chant sera entonné. Ce chant en cours d'écriture nait d'une autre série de discussion entamée au printemps 2018, non plus à propos du désespoir mais de ce qui nous fait tenir face à lui, au quotidien.

\section{Une approche éthique ancrée physiologiquement}

Avec le triptyque, il s'agit de relier, entre matière, geste et discours, un ensemble de performances engageant le fonctionnement de nos organes internes - notamment reproducteurs - avec une entité beaucoup plus large : la nature assimilée au monde comme totalité finie dans laquelle nos existences se trouvent engagées. Comme évoqué dans la première partie, la physiologie (du grec phusis, la nature, et logos, l'étude, la science) étudie le fonctionnement interne des organismes vivants. Le terme phusis définit pourtant historiquement la nature de façon bien plus générique, en tant que processus actif de naissance et déploiement. Dans cette troisième partie nous exposerons en quoi les performances engagent, à partir d'une mise en 
scène de processus qui se déploient depuis le corps féminin, une réflexion à l'échelle de la biosphère, pour nous mettre en situation, ou en état, d'agir collectivement vis-à-vis des problèmes écologiques contemporains.

\subsection{Libations et offrandes : écoulement et don de soi}

Les liquides qui s'écoulent du corps féminin sont constitués en offrande par les performances. Faire offrande de nos fluides corporels apparaît dans ce contexte comme un don. Un geste qui permet de montrer - sans forcément la « représenter »- une forme relationnelle qui est celle d'un lien nourricier réciproque entre humains et autres êtres vivants. Nous faisons référence ici à l'usage indicatif du langage, relatif à des propositions qui ne représentent rien mais nous montrent quelque chose qu'elles ne peuvent exprimer. On peut également plus simplement parler de langage poétique (voir à ce sujet Hadot [44] en référence à Wittgenstein).

Si les rituels apparentés aux sacrifices ont soulevé l'intérêt des anthropologues dès les prémices de la discipline, avec par exemple le travail fondateur d'Hubert et Mauss [45] «Essai sur la nature et la fonction du sacrifice », on trouve peu de réflexions générales à leur propos à l'époque contemporaine. Dans leur sens, le plus général, les libations consistent à verser sur le sol ou sur un objet un liquide, de manière à s'adresser à une entité sacrée, à Dieu ou à un ancêtre. Il peut s'agir de vin ou de l'huile, de bière mêlée de miel, ou encore, de lait. Faire un vœu, demander protection ou encore, se purifier de la faute commise lors d'un sacrifice sanglant. Fait largement répandu, le sacrifice revêt en fonction des contextes historiques et culturels des sens évidemment très divers. Nous pouvons à ce titre nous sentir libre d'en créer un relatif aux problèmes particuliers auxquels nous avons à faire face, ici et aujourd'hui.

Le point commun de base des offrandes, sacrifices, prières et libations est d'installer une communication entre monde profane et monde sacré, de s'adresser à l'invisible. Ici, pour prendre le cas du premier rituel, Galalithe, l'invisible se trouve tout simplement sous nos pieds. 
Ce que nous avons à honorer consiste en une fertilité qui n'est permise que par l'activité constante d'un ensemble d'êtres vivants en interaction, la faune du sol. Il s'agit donc de réexaminer la question de la fertilité en installant une continuité matérielle et symbolique entre nos propres corps et le sol, entre nos fluides et les éléments. Donner en offrande une partie de son propre corps : ici un fluide corporel nourrissant, relève à la fois du don et de l'offrande, c'est-à-dire du remerciement. Il s'agit d'un jeu avec le sacrifice de soi, propre à «jouer » l'insertion des corps humains dans les cycles du monde vivant.

Concernant la remise en contexte du choix des fluides utilisés, il serait hors du propos de cet article de produire un panorama conséquent de l'utilisation du lait, du sang et des larmes dans les pratiques rituelles. Nous ferons seulement ici référence à deux ouvrages qui donnent quelques indications sur la manière dont les fluides corporels féminins ont pu être mobilisés pour produire des récits à propos de la fertilité en contexte européen chrétien : nous faisons référence à cet ensemble comme contexte historico-culturel d'émergence de l'approche scientifique du monde, décrite en première partie.

Salvadore d'Onofrio [46] s'est intéressé aux rituels impliquant le lait et le sang en Italie du Sud. Ces pratiques, encore vivantes, dessinent un jeu avec la reproduction, la naissance et la mort, dans une conception des rapports de genre marqués par la domination masculine : la comparaison entre lait maternel et semence masculine y apparaît en particulier, en référence à Aristote, de nature à dévaloriser les produits du corps de la mère par rapport à ceux du corps du père. Gilles Tétart [47] s'est pour sa part penché sur les références au miel, dit aussi "sang des fleurs" dans l'imaginaire chrétien européen médiéval. Ce fluide, produit d'une régurgitation des abeilles, ne transite pas par l'intérieur du corps. Inscrit dans un jeu complexe d'analogies avec les liquides reproducteurs (sang, lait et sperme) il apparait comme un symbole d'une fertilité qui se passerait de la chair féminine. Par ailleurs, coulant des plaies des saints au moment de leur mort, il symbolise également la vie éternelle par annulation de la putréfaction des corps. 
Le jeu avec les fluides vise par ces deux exemples à mettre en avant une transcendance acorporelle, qui passe par une mise en suspens des cycles de la vie et de la mort, et plus spécifiquement par une dépréciation ou une annulation de la part féminine de la reproduction humaine.

En réaction, le triptyque cherche au contraire à souligner l'engagement du corps féminin, comme puissance créatrice et destructrice, dans les cycles en question et à les réhabiliter dans le même geste. En effet l'expérimentation met en relation deux plans : première dimension, l'insertion des existences humaines dans les cycles du monde vivant, à l'écoute de la circulation des flux de matière, d'information et d'énergie supports de vie, à la surface de la terre, au sens de planète terre. La seconde dimension renvoie à un imaginaire renouvelé de l'engendrement, en lien avec la question de la filiation, de la parenté. Celui-ci reprend pied dans l'histoire longue des évolutions des figures féminines associées à la terre, mais dans un contexte où la féminité se réinvente. Au-delà de la maternité, cela amène donc à une référence beaucoup plus large à l'action de donner naissance ou de favoriser la vie. Enfin nous prenons appui comme intermédiaire, sur les éléments pris au sens symbolique, que nous rassemblons sous quatre entités familières à l'histoire européenne : le feu, la terre, l'eau, et l'air.

Ce jeu d'analogie s'appuie sur une figure ternaire, en décalage par rapport à la série d'oppositions binaires asymétriques entre masculin et féminin, haut et bas, sec et humide, chaud et froid, etc. [48]. Le lait maternel, le sang menstruel, les larmes de tristesse et de deuil engagent trois lieux du corps : les seins, le sexe, les yeux. Ils entrent par la suite en résonnance avec trois éléments, milieux, et trois âges de la vie.

Le sang est pour l'eau, vers l'avant, à une rivière qui se répand dans l'océan. L'âge correspondant est celui de la jeunesse, du passage, une voix en puissance. 
Le lait est pour la terre, vers le bas, à un sol, qui accompagne fertilité et régénération. L'âge correspondant est la maternité, et plus généralement, la créativité en acte.

Les larmes sont pour l'air, vers le haut : elles évoquent l'évaporation, le deuil, départ et changement d'état du monde, nos désespoirs mêlés. L'âge correspondant est la vieillesse et le déclin des forces. Le feu n'apparait pas directement, dans la mesure où il n'est pas à la fois un élément et un milieu à part entière, mais plutôt, un phénomène. Sur le plan physique, il renvoie aux étoiles, aux volcans, aux éclairs, à la combustion. Concernant la fabrication des offrandes, il est au cœur de chaque manipulation en tant qu'agent transformateur. C'est le phénomène qui fait muter le lait maternel en ersatz d'albâtre, une matière transparente, dure et cassante, c'est lui qui permet de faire muter le sang menstruel en pépites métalliques et enfin, les larmes en sel.

\subsection{Des réactions en clair-obscur}

Les expériences menées dans le triptyque sont ouvertes, dans la mesure où elles servent de support à des discussions avec différents types de publics, qui participent à leur évolution.

Lors de la première exposition de Galalithe au domaine départemental de Chamarande, l'installation s'est révélée perturbante. Si le lait maternel est un produit dont il est peut-être plus facile de parler sans pudeur aujourd'hui qu'il y a quelques décennies avec le retour en grâce de l'allaitement, il renvoie néanmoins tout de même à l'intimité féminine. Nous avons donc été attentives aux rapprochements qui ont surgi chez nos interlocuteurs. Ceux-ci nous ont surpris ${ }^{8}$. En dehors du lait lui-même, le thème le plus régulièrement abordé fut celui de la mort et de la

\footnotetext{
${ }^{8}$ Les discussions avec le public ont eu lieu lors de l'inauguration et de la clôture de l'exposition ainsi que d'une visite guidée que nous avons organisée. Le public était essentiellement composé d'habitants du secteur.
} 
décomposition des corps. Par extension, donner quelque chose de son corps au sol évoque l'enterrement des morts et leur dégradation des chairs par l'entremise de la faune du sol. Les larves de mouche, autres êtres vermiformes, sont ainsi entrées en scène à plusieurs reprises, comme plus à même de consommer ce que nous avions à donner. Les pratiques de traitement des corps chez les chrétiens ont aussi été évoquées : le cercueil de bois dur et le tombeau de pierre ont vocation à annuler l'idée de putréfaction, qui entre en contradiction avec la résurrection de la chair qui donne accès à la vie éternelle, condition transcendante en rupture avec les cycles reproductifs...à l'instar de la naissance du Christ sans le recours au principe féminin. Ce déni de la pourriture passe également par l'embaumement des corps. La dégradation des cadavres est pointée du doigt comme un problème matériel, puisque l'embaumement les rend impropre à la consommation pour les décomposeurs et même, toxiques... Notre propre alimentation, les antibiotiques, et la dégradation de notre microbiome ne facilite d'ailleurs pas non plus le travail de la pourriture, comme l'ont souligné plusieurs visiteurs. Ainsi la discussion passe du lait maternel aux cadavres et à leur décomposition. C'est cette référence à la mort dans les échanges menés dès le début de l'expérience qui nous ont incité à vouloir éviter d'opposer la célébration et l'apaisement à une dimension plus sombre que nous avons choisi d'endosser en nous intéressant au sang.

Or l'évocation du sang, lors de la présentation publique de Selenhydre, a ramené à l'inverse à la question du lien nourricier. Ainsi, nous avons eu l'occasion de rencontrer une femme - cas très particulier sans doute, qui procédait parfois de façon solennelle à des offrandes de sang menstruel à ses plantes d'appartement «pour ne pas le gâcher », mais surtout selon ses dires, «par amour » pour ces dernières. Ce sang-là fait ordinairement l'objet d'un dégoût profond, d'une façon assez analogue aux cadavres : quel renversement que d'en faire un aliment à offrir ! Un autre participant souligne que donner aux plantes ce dont on a plus besoin, que cela soit des restes d'aliments ou encore, n'importe quel déchet corporel (urine, matière fécale, ongles, 
cheveux), peut être considéré selon une certaine perspective, comme une offrande ou du moins, un don.

Enfin, l'évocation des larmes a été identifiée à un support de compréhension de la cyclicité des désastres écologiques. Il est question de réciprocité, comme le souligne l'une des participantes à l'expérience l'Appel aux larmes : on ressent bien que «ce qu'on lui fait à la terre on se le fait à nous même parce que nous sommes une seule et même chose, nous en faisons partie ». Ainsi l'eau des larmes qui lave les pieds ou les mains est aussi à un autre moment, celle que l'on boit. L'eau entre en nous et nous baigne, de l'utérus à l'océan. Comment faire confiance à celui ou celle qui vous offre à boire, au milieu qui vous nourrit ? Dans un contexte où la terre est sommée de donner continuellement et jusqu'à l'asphyxie complète, il devient loisible de penser que ses cadeaux se révèlent, à terme, empoisonnés.

\subsection{Inventer des rituels, se mettre en situation}

Comme évoqué au début de ce propos, l'expérience du triptyque se place sur le plan de la subjectivité. Nous nous référons à la définition qu'en donne Guattari [49] comme une propriété qui ne se trouve pas logée à l'intérieur des individus mais les traverse, les dépasse et les relie entre eux et au monde. Si la forme que nous avons choisie est le rituel, c'est dans l'intention de participer à un déclenchement dans le présent de ce qu'il nomme une « coupure-bifurcation » propre à intervenir sur le plan subjectif, via la production d'une nouvelle narration : notre invention amène, nous le constatons, quelque chose qui s'installe dans la durée et qui nous échappe...

Procéder par la fabrication rituelle consiste en une performance poétique qui fait appel à des structures symboliques largement partagées dans un contexte culturel donné, le nôtre, en l'occurrence. En écho des pratiques traditionnelles de manipulation dite magique des fluides 
corporels, ces élaborations nous mettent en rapport avec l'invisible, le caché, l'occulte (i.e. l'occulté, ce à quoi nous n'avons pas accès dans la pratique courante et quotidienne). Par ailleurs, cela permet de rétablir un lien rompu dans le langage par la séparation entre prose et poésie, art et science, approche sensible et rationnelle du monde [50]. Cela permet enfin de comprendre par nos propres corps mis en jeu, mais aussi d'affirmer les interdépendances qui nous lient à nos milieux de vies. Il ne s'agit pas de mettre en évidence ou de démontrer mais de procéder par l'expérience pour découvrir un mode de connaissance mieux lié aux perceptions directes, mieux incarné, à développer une autre attention, à la fois sensible et éthique, au sens de Jan Masschelein.

Cette logique se retrouve dans le processus de production des coupes, traces matérielles du processus du triptyque. Ainsi au lieu de définir à l'avance selon des procédés connus, les formes produites à partir des fluides des rituels, nous avons choisi de laisser parler la matière (figure 6). Il a fallu la travailler, l'adapter, agir avec elle, évoluant avec ce qu'elle offrait (résistance, cristallisation, craquellement...). Par cette mise en pratique, dans notre processus même de création, le sensible retrouve son importance contre la «déprise » qu'engendre la sursophistication technique et conceptuelle. 


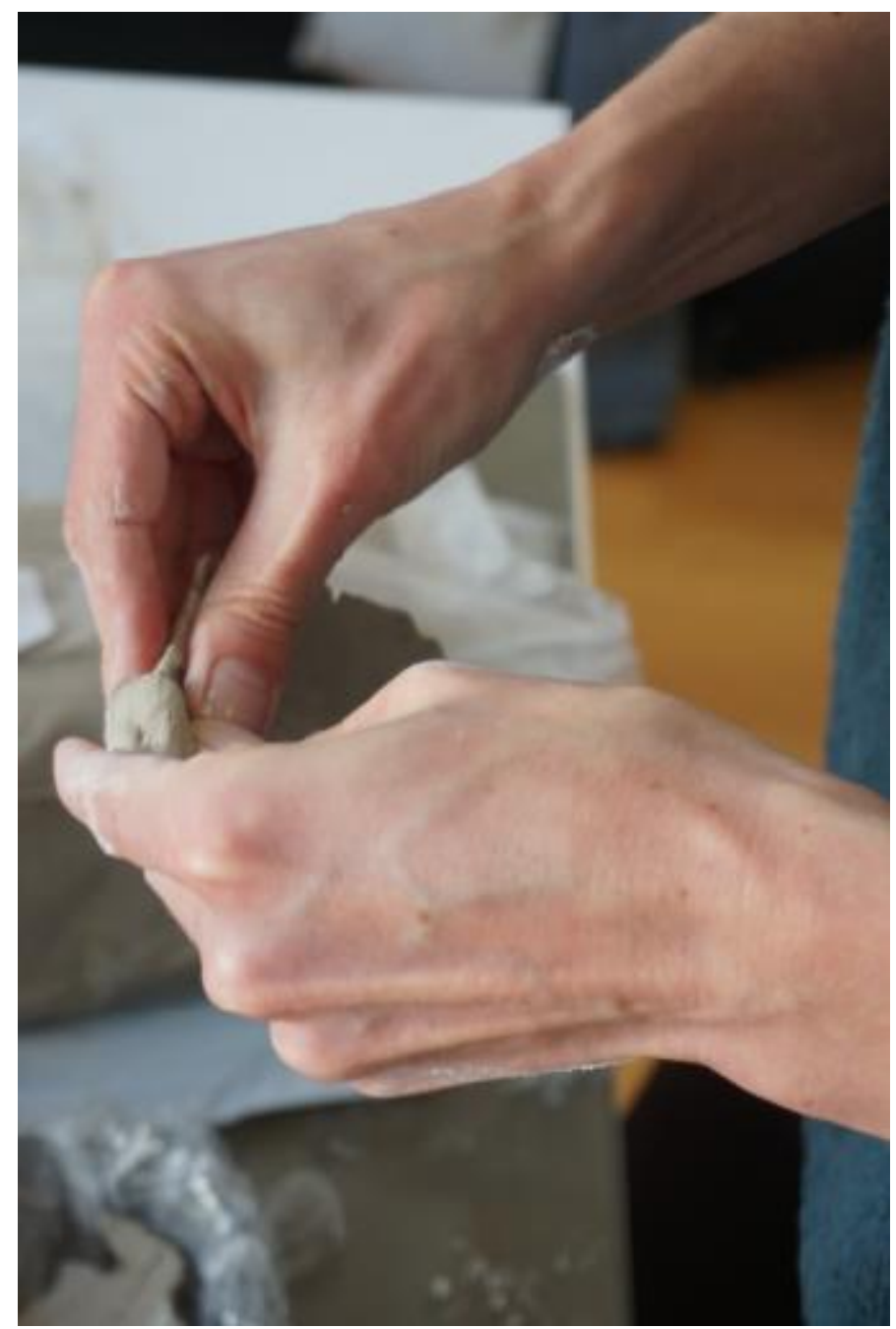

Figure 6 : Travail de l'argile pour l'Appel aux larmes, 2018 (C) Anaïs Tondeur

Nous considérons la performance ritualisée comme un outil de compréhension et d'affirmation d'un lien nourricier réciproque. Elle vise à faire sentir et comprendre les interdépendances autrement qu'en les conceptualisant, par la mise en œuvre et la mise en scène. Pourtant, inventer un rituel, n'est-ce pas une idée absurde ? Les anthropologues ont pour habitude de décomposer pour les comprendre les rituels des sociétés qu'ils étudient. L'analyse fine de la gestuelle et les discours mobilisés par exemple pour faire tomber la pluie ou fertiliser la terre, pour maintenir le monde en ordre, donnent à voir des structures sous-jacentes, des motifs qui irriguent les pratiques rituelles mais que ceux qui les mobilisent n'explicitent pas. 
En outre un rituel est un geste qui prend corps et se transmet sur le temps long, par la répétition. Pourtant il est intéressant de constater que l'invention rituelle a de longue date une vocation politique : en puisant dans l'histoire locale ou en rupture manifeste avec elle, elle s'expose dans l'espace public. Les responsables politiques s'en emparent : ainsi l'offrande de fleurs au fleuve mise en scène sur le Rhône dès les années 1920 puisait-elle dans les traditions paysannes pour nourrir l'adhésion populaire à la construction de barrages sur le fleuve [51]. Les activistes aussi ritualisent leurs performances, tels que les brigades de clowns activistes contemporaines [52], les femmes de la Wicca qui s'adonnent à des danses spirales sous la brûlure des gaz lacrymogènes lors des manifestations altermondialistes [53]. En explicitant les motifs mobilisés, nous procédons finalement à autre chose que ce que nous avions annoncé : l'intérêt et l'efficacité de la pratique ne se situe pas directement dans le bricolage symbolique qui nous sert de prétexte comme Michael Houseman [54] l'a par exemple montré pour les cérémonies de célébration des «ménarches », ou premières règles, qui s'inventent depuis les années 1970 et se célèbrent en famille ou entre amies. Autrement dit de façon plus imagée, cette fabrication nous sert de clé pour ouvrir un coffre - mais nous ne savons pas ce qui se trouve dedans. Les performances, leurs récits et les débats qui les accompagnent permettent avant tout la production de situations, où nous jouons sur le fil en tâchant d'éviter le risque de dépolitisation. En agissant poétiquement, nous cherchons au contraire en ouvrant de nouvelles possibilités d'agencement entre corps, matière et langage à assumer une critique des rapports de production. Il convient de créer une situation qui donne le pouvoir de faire penser, sentir, agir, en dehors des cadres présupposés, comme l'avance notamment la philosophe Isabelle Stengers [55] à propos de sa réflexion sur « la sorcellerie capitaliste »- précisons encore que le terme est bien ici employé pour expliciter le plus sérieusement du monde des mécanismes socio-politiques au cœur des rapports de production.

\section{Conclusion : jouer avec la matière mouvante}


Le triptyque propose d'appréhender la biosphère ou encore le cosmos terrien, compris comme entité finie où tout circule ${ }^{9}$. Mais ceci posé, reste encore une question : Que faire, dans ce contexte, de l'envie d'infini, si structurante de la condition humaine ? Sur le plan existentiel la quête reste, effectivement, intacte et nécessaire, et à repenser dans sa tension avec la question biopolitique [56]. La prise de conscience et la preuve de la finitude planétaire posent des limites, sur le plan matériel, à ce que la puissance technique peut faire. La conquête spatiale et l'espoir de vie éternelle proposée par le trans-humanisme cherchent éperdument, y versant tous ses efforts, à gommer ces limites, sans encore y parvenir. Voilà une porte qui se ferme.

C'est peut-être pour le mieux si l'on considère que chaque individu est constitué de la diversité des relations auxquelles il participe. Car alors, détruire, manquer de considération envers ce dont nous sommes constitués, ces relations avec les autres vivants, c'est également et tout autant nous manquer de respect à nous même. C'est aussi appauvrir notre existence, au sein d'un monde de plus en plus petit, bien qu'augmenté de l'ensemble de nos productions, machines et textes. Productions techniques et culturelles. Ces machines et ces textes, ces images et ces bâtiments, par clôture de l'humain sur lui-même. Ce qui pouvait apparaitre comme un affranchissement, un arrachement à la glèbe des champs et à la boue des chemins apparait selon cette perspective comme un autre enfermement. Il est possible de nier les relations avec les autres vivants ou de les délaisser, de les effacer, de ne plus faire référence qu'à nous même, comme le souligne David Abram [57], dans son analyse de la mutation des liens entre langages humains et environnements au cours de l'histoire de l'écriture. Néanmoins, poursuivre selon cette logique de toute-puissance engage sur le chemin d'une solitude étroite et désolée, vers, en fait, le néant.

\footnotetext{
${ }^{9}$ Nous pourrions aussi dire Gaïa, comme le font aujourd'hui plusieurs philosophes de premier plan tels que Bruno Latour ou Isabelle Stengers mais préférons, par prudence, nous en abstenir.
} 
Loin de conquérir l'espace infini des étoiles, traversant le vide intersidéral pour enfin s'arracher au berceau nous avons peut-être en gage de nous réconcilier avec la terre dont nous sommes nés, en considérant ce retour comme un plongeon vers d'autres avenirs - à partir de l'attention portée aux autres êtres : pour une rencontre de l'altérité, un vertige des espace-temps recomposés, des existences mêlées, en somme un autre infini, inlassablement réitéré, celui de la complexité.

\section{Remerciements}

Les membres du collectif Chaoïde - Alan Vergnes, Germain Meulemans, Yesenia Thibault Picazo - ainsi que Nathalie Blanc \& Maëva Blandin pour le Laboratoire de la culture durable ; Lauranne Germond et Loïc Fel pour le Laboratoire de la culture durable ; Joanne Clavel pour notre travail commun autour de l'Appel aux larmes ; les visiteurs de l'exposition SOLS FICTIONS, les organisateurs des différents évènements qui ont accompagné la création du triptyque et participants à ces expériences : présentation LASER, Annick Bureaud ; séminaire «méthodes sensorielles et visuelles en anthropologie » EHESS (en particulier Florencia Muñoz Ebensperger et Léo Nguyen Van Thé) ; Perig Pitou et les membres du colloque « La vie à l'œuvre » au Musée de la Chasse et de la Nature ; les participants à la performance L'Appel aux larmes au Centre Tignous pour l'Art Contemporain (en particulier Hélène, Laure, Marianne, Sidonie \& Cécile) 